\title{
ORIGINAL RESEARCH

THE EFFECTIVENESS OF EMG BIOFEEDBACK ON
HAND FUNCTION IN SUBJECTS WITH STROKE

${ }^{1}$ S. Sethana

${ }^{2}$ V. Sri Kumari

${ }^{3} \mathrm{~K}$. Madhavi

\section{ABSTRACT}

Introduction: Stroke is an event caused by the interruption of the blood supply to the brain, usually because a blood vessel bursts or blocked by a clot. Biofeedback can be defined as the technique of using equipment usually electronic to reveal to human beings about some of their internal physiological events normal and abnormal in form of auditory and visual signals.

Method: The stroke patients diagnosed by neurologist were recruited from physiotherapy department and inpatients from neurology and general wards of SVIMS hospital, Tirupathi Andhra Pradesh. In the present study 30 subjects were randomly assigned to 15 experimental and 15 control groups. The subject was made to sit comfortably and the Surfaces electrodes were placed on Extensor carpi radialis, Extensor digitorum communis muscle belly and for 30minutes patient voluntarily contracts until signals displayed on screen for which visually and auditory cues are given. In control group placebo EMG where machine is turned away $\mathcal{E}$ has no cues. Both groups received CONVENTIONAL PHYSIOTHERAPY; for 30 minutes at a Frequency: 1 hour per day for 5days in a week, for 6weeks.

Results: There was statistically significant $(\mathrm{p}<0.05)$ improvement in both variables from baseline to 6thweek in experimental group compared to control group.

Conclusion: Our study demonstrates the potential benefits of EMG BF in improving hand function in subjects with stroke.

Keywords: Electromyographic Biofeedback (EMG BF), Box and Block Test (BEBT)

Received $15^{\text {th }}$ July 2014, revised $14^{\text {th }}$ August 2014, accepted $26^{\text {th }}$ August 2014

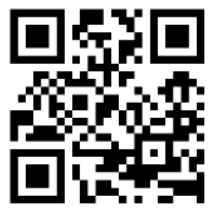

WwW.ijphy.org
DOI: 10.15621/ijphy/2014/v1i4/54557

\footnotetext{
${ }^{2}$ Associate Professor

${ }^{3}$ Professor and Principal

College of Physiotherapy,

Sri Venkateswara Institute of Medical Sciences,

Tirupati, Andhra Pradesh, India.
}

\section{CORRESPONDING AUTHOR}

\section{${ }^{1}$ S. Sethana}

MPT Post Graduate Student, College of Physiotherapy, Sri Venkateswara Institute of Medical Sciences, Tirupati, Andhra Pradesh, India. e-Mail: sethana.physio@gmail.com 


\section{INTRODUCTION}

According to WHO stroke is caused by the interruption of the blood supply to the brain, usually because of either a blood vessel bursts or is blocked by a clot. This cut off the supply of oxygen and nutrients, causing damage to the brain tissue. ${ }^{1}$ It is the third commonest cause of mortality ${ }^{2}$ and the fourth leading cause of disease burden. ${ }^{3}$ The most common symptom of a stroke is sudden weakness or numbness of the face, arm or leg, most often on one side of the body. Other symptoms include: Loss of speech, or difficulty talking, Dimness or loss of vision, Unexplained dizziness, especially when associated with any of the above signs, Unsteadiness or sudden falls, Headache (usually severe and of sudden onset), Confusion. The effects of a stroke depend on the part of the brain which is injured and how severely it is affected. A very severe stroke can cause sudden death. Recovery of motor function after stroke will be completed within 3-6 months after onset but in can continue for months or years. ${ }^{4,5}$

Upper limb weakness is frequent after stroke ${ }^{6}$ and its recovery is limited. At 6 months post stroke only approximately $12 \%$ of stroke population are able to regain full arm and hand function. ${ }^{7}$ Poor recovery of hemiplegic upper limb is due to the inappropriate and inadequate physical therapy ${ }^{8,9}$ along with noninvolvement of affected upper limb in the regular activities of daily living which is likely to result in further weakness, and connective tissue changes like contractures also occur which further results in learned nonuse syndrome ${ }^{10}$ sensory loss ${ }^{11}$ and loss of cortical representation.$^{12}$ Therefore recovery of upper limb function after stroke follow a predictable manner and time course of it is variable.

Learned non-use syndrome is a phenomenon observed in patients with stroke characterized by failure to use the affected upper limb following sensory, motor, loss with attention being directed towards unaffected contralateral extremity. In neuroplasticity, there are 2 major advances marked in neurological rehabilitation over the past 2 decades. Demonstration of the pernicious nature of inactivity that generates tenacious learned nonused underpinned by cortical reorganization ("learned non-use") and demonstration of neural reorganization after a cerebral lesion under the effect of use ("use-dependent") and consequently, the possibility of "driving plasticity."

According to the theory in LEARNED NON-USE there are repeated disappointments in attempts to use the affected arm leading to negative reinforcement of using the affected arm. Therefore the individual learns, not to use the affected $\operatorname{arm}^{13,}$ 14

The motor recovery is fastest during early weeks. Restoration of function during initial six weeks is attributed to various factors like: dendritic sprouting, synaptogenesis, restoration of axonal transport, remyelination, resolution of diachisis, alterations in neurotransmitters and bilaterality of brain functions.

Schimdtz defined motor learning as a set of process associated with practice or experience leading to relatively permanent changes in capability for responding. Primary factors influencing motor control which includes Stage of learner, type of task, feedback and practice. Feedback is of two types, they are intrinsic and extrinsic; extrinsic includes knowledge of results and performance.

1. Open-loop-control :- preplanning feed forward

2. Closed-loop-control:- using continuous external feedback until motor skills develop sufficiently.

Bio-feedback is a technique of using equipment usually electronic to reveal to human beings about their internal physiological events normal and abnormal in form of auditory and visual signals, To teach them to manipulate these otherwise involuntary or unfelt events by manipulating the displayed signals.(SUSAN M BLACKMORE WILLIAMS, WOLF). Biofeedback usually involves measurement of a target biomedical variable and relaying it to the user using one of two strategies; 1. Direct feedback regarding the measured variable, where a numerical value is displayed on a screen. 2. Transformed feedback regarding the measured variable, where the measurements are used to control an adaptive auditory signal, visual display or tactile feedback method. The biofeedback measurements which are frequently used in physical rehabilitation can be categorized as being either physiological or biomechanical. ${ }^{15}$

\section{MATERIALS AND METHODS}

- Source of data: participants were recruited from outpatient physiotherapy department and inpatients in neurology and general wards in SVIMS hospital.

- Study design: A randomized controlled trail, prospective experimental design with pre-test and post-test design.

- Sample design: The participants were undergone simple randomized sampling by giving a block number to each patient $\mathcal{E}$ randomized into two groups that is intervention and control group.

- Study sample: 30 stroke subjects who are fulfilling with inclusive criteria. 
- Materials used: Hand gripper, Box and block test kit, Electrical stimulator, Stop watch, Myomed (EMGBF) Machine.

\section{CRITERIA FOR THE STUDY:}

\section{Inclusion criteria:}

- Stroke subjects with first episode of infarct or hemorrhage stroke confirmed with CT and MRI scan by a neurologist.

- Gender: both male and female stroke patients were included in the study

- Stroke subjects with right hemiparesis,

- Stroke subjects whose age between 45-70yrs,

- Stroke subject who have spasticity with Modified Ashworth Scale grade 1, 1+

- Stroke subjects who are in stage 2 and 3 of Brunnstorm stages of recovery

- Stroke subjects whose cognitive abilities is good with MMSE score $>24$.

Exclusion criteria:

- Stroke subjects who are in Flaccid stage of hemiplegia,

- Stroke subjects who have spasticity is > or $=2$ of MAS grade

- Stroke subjects with Recurrent strokes,

- Stroke subjects with any Visual impairments

- Stroke subjects with perceptual deficits,

- Subjects with any significant upper limb musculo-skeletal or neurological deficits other than stroke, and any unstable cardiovascular status.

- Stroke subjects with Dementia and

- Stroke subjects with Sensory aphasia.

\section{METHODOLOGY}

All the Stroke subjects who are diagnosed as infarct or haemorrhagic stroke based on CT or MRI scan were selected on the basis of inclusion criteria; The subjects participated in this study voluntarily after the signing the consent form. The demographic data, baseline measurements were collected from both the groups and the purpose of the study was explained to all the subjects.

EXPERIMENTAL GROUP: The subject was made to sit comfortably in a chair with forearm pronated and fully supported and wrist was in neutral position with no muscles stretched unduly; once electrodes were placed on either ECU, ECR, EDC muscle belly. (Wrist extensors which are not involved in either synergy). And assessed any EMG activity while subject is at rest, the patient was motivated to voluntarily extend the wrist until signals displayed on screen and further motivation was provided to the patient by the machine with visual cues in the form graphs and auditory cues in the form of sound. Treatment duration was 30minutes with proper rest intervals in were incorporated in between to prevent the undue fatigue.

Figure: Myomed Machine

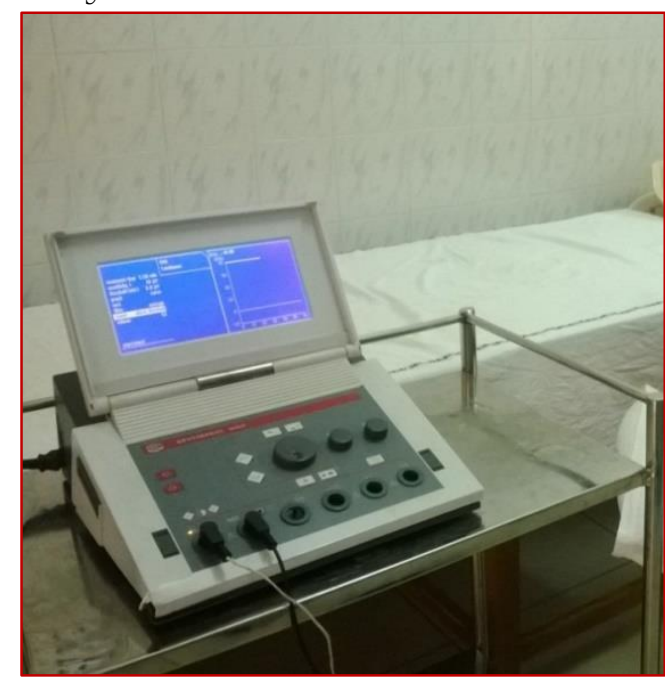

EMG Biofeedback Machine parameters:

- 2 Active electrodes: are placed $2 \mathrm{~cm}$ apart on the desired motor point and the Ground electrode: between the two active electrodes to prevent the unwanted artifacts

- Sensitivity: 20-100 microvolts

- Thresh hold EMG is peak value felt by the subject usually set at $50 \mathrm{Uv}$

- Graph: Curves

- Filter: average

- Sound: beep sound heard above threshold

- Volume: adjusted until sound is heard.

- Technical aspects: reliability and validity of the equipment is established

- Treatment duration: 30 minutes.

CONTROL GROUP: In control group a Placebo EMG is used which was switched 'on' but the machine is turned away from the patient and volume was also muted so that no verbal and auditory cues were provided to the patient.

\section{CONVENTIONAL THERAPY:}

It is given for both the group for a period of 30 minutes. It includes

- Electrical stimulation for weaker wrist extensors of the affected upper limb.

- Prolong icing and stretching's to spastic group muscles of the affected upper limb.

- Strengthening exercises to affected upper limb, using weight cuffs.

- Free exercises and active movements to the affected upper limb in sitting position.

- Weight bearing exercises to the affected upper limb: 
- Hand grip exercises: using hand gripper with an adjustable resistance.

- Gross motor training for the affected hand in order to aid for:

\section{STATISTICAL ANALYSIS}

Statistical analysis was done using SPSS 20 version software. For this purpose the data was entered into Microsoft Excel spread sheet, tabulated and subjected to statistical analysis.

Out of 30 subjects, 15 were randomized into experimental group and 15 were randomized into control group. All the subjects completed the entire study protocol as defined by 6 weeks in training sessions.

Pretest and posttest values of box\& block test were measured for evaluation of basic manual dexterity of upper limb and pre Eposttest values of EMG potentials for measurement of muscle activity.

To compare the pretest $\&$ posttest treatment effect within the group paired test was used, and to compare the pretest $\mathcal{E}$ posttest treatment effect between the groups unpaired t-test was used.

RESULTS:

Table: 1 Demographic $\mathcal{E}$ clinical characteristic of samples at baseline.

\begin{tabular}{|c|c|c|c|}
\hline VARIABLE & $\begin{array}{l}\text { CONTROL GROUP } \\
(\mathrm{n}=15)\end{array}$ & $\begin{array}{l}\text { EXPERIMENTAL GROUP } \\
\qquad(\mathrm{n}=15)\end{array}$ & p VALUE \\
\hline Sex & $9 \mathrm{M} / 6 \mathrm{~F}$ & $10 \mathrm{M} / 5 \mathrm{~F}$ & $\begin{array}{l}X^{2}=3.767 \\
P=0.0877\end{array}$ \\
\hline Age (mean) years & 53.73333 & 54.26667 & 0.501 \\
\hline Side of paralysis & Right & Right & - \\
\hline $\begin{array}{l}\text { MMSE SCORE } \\
\text { (score normal value 24-30) }\end{array}$ & 26.73333 & 26.46667 & 0.603 \\
\hline
\end{tabular}

$\mathrm{M}=$ male $\mathrm{f}=$ female $; \mathrm{MMSE}=$ minimental status examination

Demographic characteristics of subjects with stroke is shown in Table-1. The statistics shown that there were no statistical significance between control and intervention group.

\section{CONTROL GROUP:}

Table 2: Analysis of control group with pre and post intervention

\begin{tabular}{|l|c|c|c|c|c|c|}
\hline \multicolumn{1}{|c}{ VARIABLE } & N & Mean & S.D & t-value & Df & p-value \\
\hline EMG potentials $(\mu$ ) & & & & & & \\
Pre & 15 & 46.9333 & 5.049281 & 0.280765 & 14 & $<0.05$ \\
Post & 15 & 47.9333 & 11.47959 & & & \\
\hline BEBT (blocks per minute) & & & & & & \\
Pre & 15 & 10.8667 & 3.522715 & 9.057405 & 14 & $<0.05$ \\
Post & 15 & 12.9333 & 3.673587 & & & \\
\hline
\end{tabular}

S.D = Standard deviation, $\mathrm{Df}=$ degrees of freedom; $\mathrm{N}=$ number of subjects

EMG potential: pre $\&$ post values were compared by using paired sample t-test. The p-value was $<0.05$ which shows there was extremely significant difference. The t-test value was
0.280765 with 14 degrees of freedom. It was observed that the post intervention had shown significant impact on the subjects. 
Chart: 1 Graphical representation of pre $\&$ post values of EMG potentials in control group:

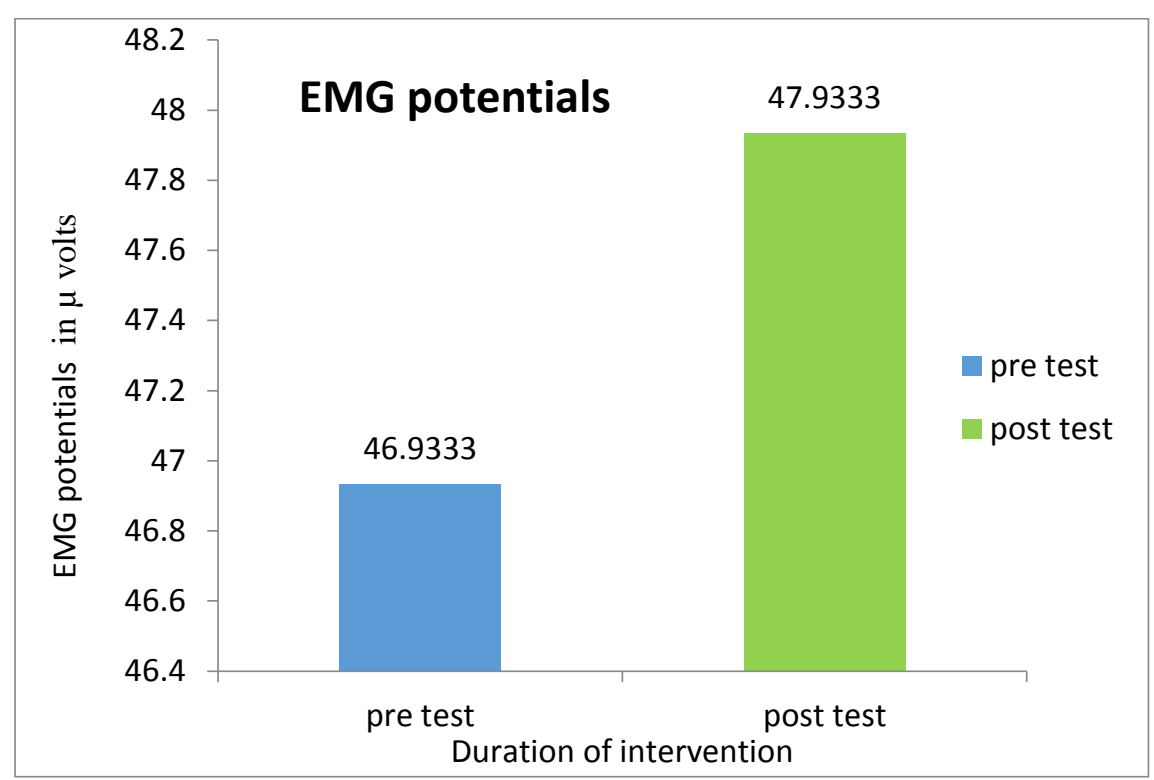

There was a significant difference between pre $\mathcal{E}$ post intervention of control group in EMG potentials on hand function in subjects with stroke.

B \& BT: Pre \&post values were compared by using paired sample t-test. The $\mathrm{p}$-value was $<0.05$ which shows there was extremely significant difference. The t-test value is9.057405with 14 degrees of freedom. It was observed that the post intervention had shown significant impact on the subjects.

Chart: 2 Graphical representations of pre $\mathcal{E}$ post values of BEBT in control group:

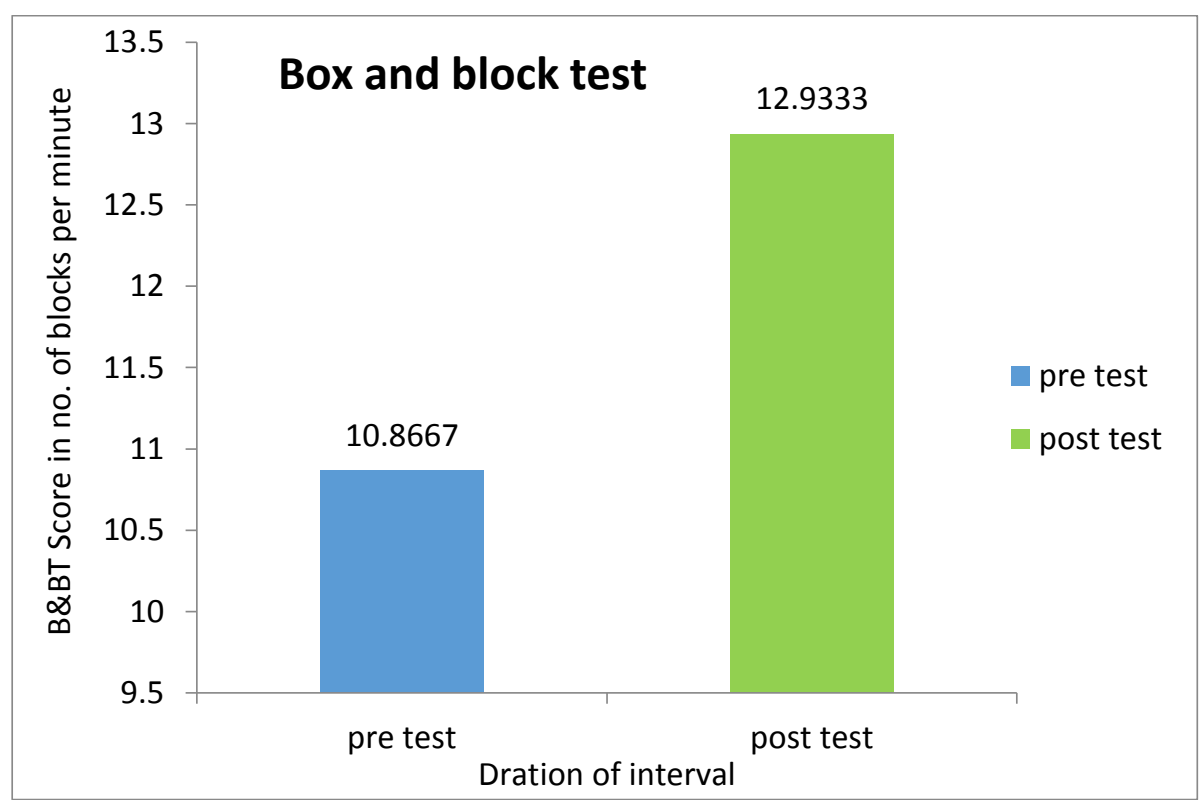

There was a significant difference between pre $\mathcal{E}$ post intervention of control group in BEBT on hand function in subjects with stroke.

\section{EXPERIMENTAL GROUP}

EMG potential: pre $\&$ post values were compared by using paired sample t-test. The p-value was
$<0.05$ which shows there was extremely significant difference. The t-test value was8.2794with 14degrees of freedom. It was observed that the post intervention had shown significant impact on the subjects. 
Table: 3 Analysis of experimental group with pre $\mathcal{E}$ post intervention:

\begin{tabular}{|c|c|c|c|c|c|c|}
\hline Parameters & $\mathbf{N}$ & Mean & S.D & t-value & Df & p-value \\
\hline $\begin{array}{l}\text { EMGpotentials }(\mu V) \\
\text { Pre } \\
\text { Post }\end{array}$ & $\begin{array}{l}15 \\
15\end{array}$ & $\begin{array}{c}47.3333 \\
57.6\end{array}$ & $\begin{array}{c}6.768273 \\
8.00714\end{array}$ & 8.2794 & 14 & $<0.05$ \\
\hline $\begin{array}{l}\text { BEBT(blocks per minute) } \\
\text { Pre } \\
\text { Post }\end{array}$ & $\begin{array}{l}15 \\
15\end{array}$ & $\begin{array}{c}10.2 \\
18.4667\end{array}$ & $\begin{array}{l}4.887301 \\
4.897035\end{array}$ & 10.5501 & 14 & $<0.05$ \\
\hline
\end{tabular}

\section{S.D = Standard deviation, $\mathrm{Df}=$ degrees of freedom; $\mathrm{N}=$ number of subjects.}

Chart: 3 Graphical representations of pre $\mathbb{E}$ post values of EMG potentials in experimental group:

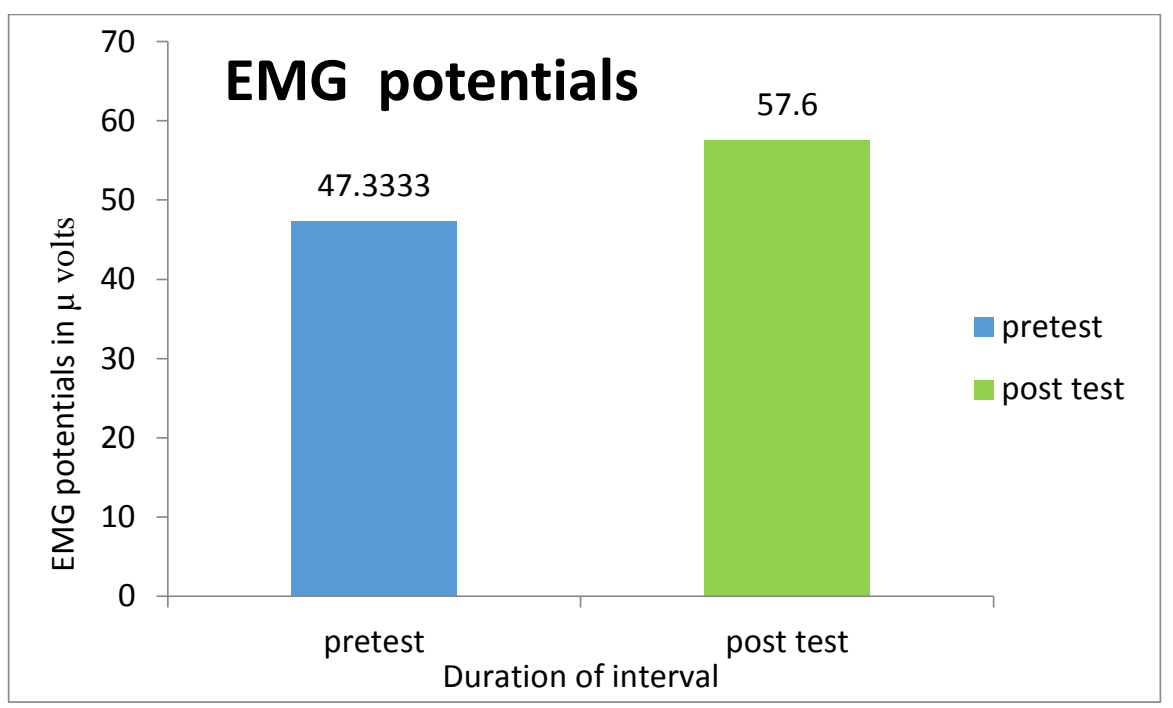

There was a significant difference between pre $\mathcal{E}$ post intervention of experimental group in EMG potentials on hand function in subjects with stroke.

B \& BT: Pre Epost values were compared by using paired sample t-test. The p-value was $<0.05$ which shows there was extremely significant difference. The t-test value was 10.5501with 14 degrees of freedom. It was observed that the post intervention had shown significant impact on the subjects

Chart: 4 Graphical representations of pre $\&$ post values of BEBT in experimental group:

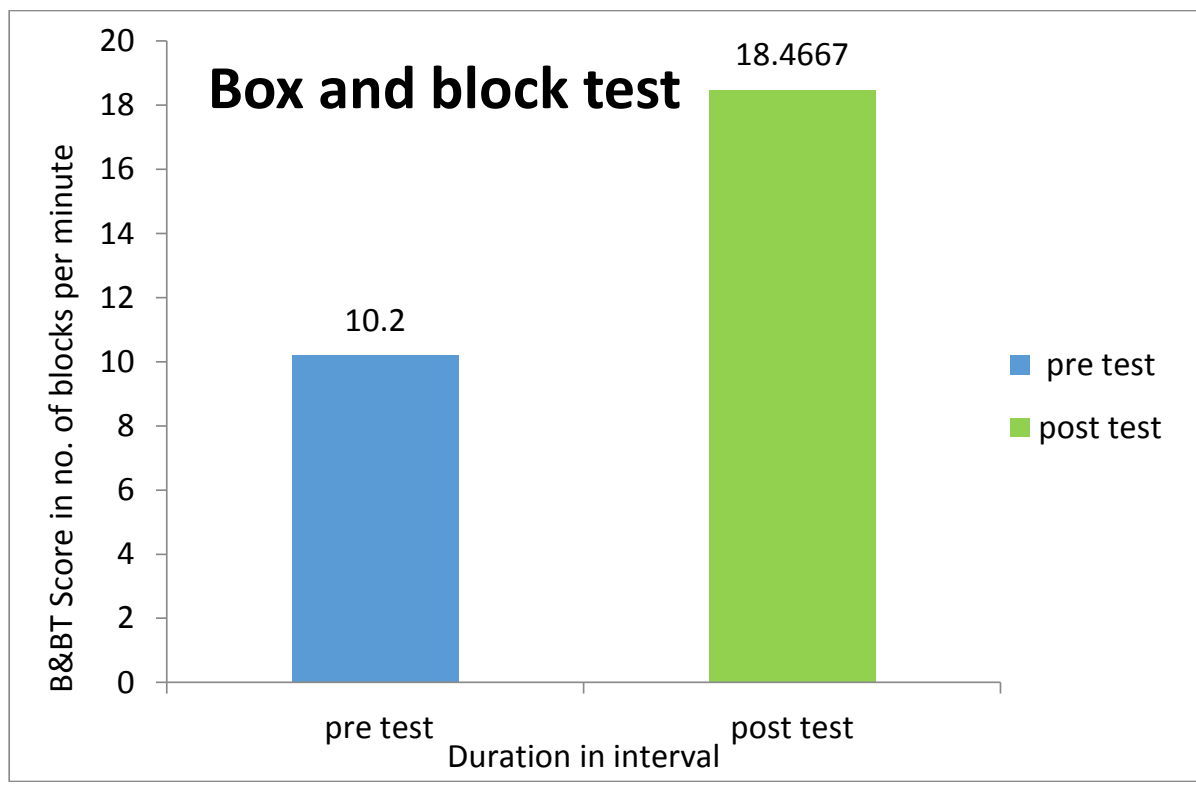

There was a significant difference between pre $\mathcal{E}$ post intervention of experimental group in BEBT on hand function in subjects with stroke. 


\section{COMPARISION BETWEEN THE GROUPS:}

Table: 4 Comparisons between the control group and experimental group:

\begin{tabular}{|l|c|c|c|c|c|}
\hline \multicolumn{1}{|c|}{ Parameters } & Groups & N & Mean & T & p value \\
\hline EMG potentials $(\mu \nu)$ & Experimental & 15 & 10.2667 & 0.854 & 0.05 \\
\hline & Control & 15 & 1.000 & & 0.05 \\
\hline $\begin{array}{l}\text { Box and block test } \\
\text { (blocks per minute) }\end{array}$ & Experimental & 15 & 8.2667 & 7.597 & 0.005 \\
\hline & Control & 15 & 2.0667 & & 0.005 \\
\hline
\end{tabular}

EMG potentials: To compare the results of between the group of control \& experimental group, unpaired t-test was selected. The p-value was 0.05 where the difference was considered very significant. The values of EMG potentials were improved in control group as well experimental group but the improvement was more in experimental group. Thus the null hypothesis is rejected and alternate hypothesis is accepted.

Chart: 5 Graphical representation of mean difference between experimental group and control group in EMG potentials:

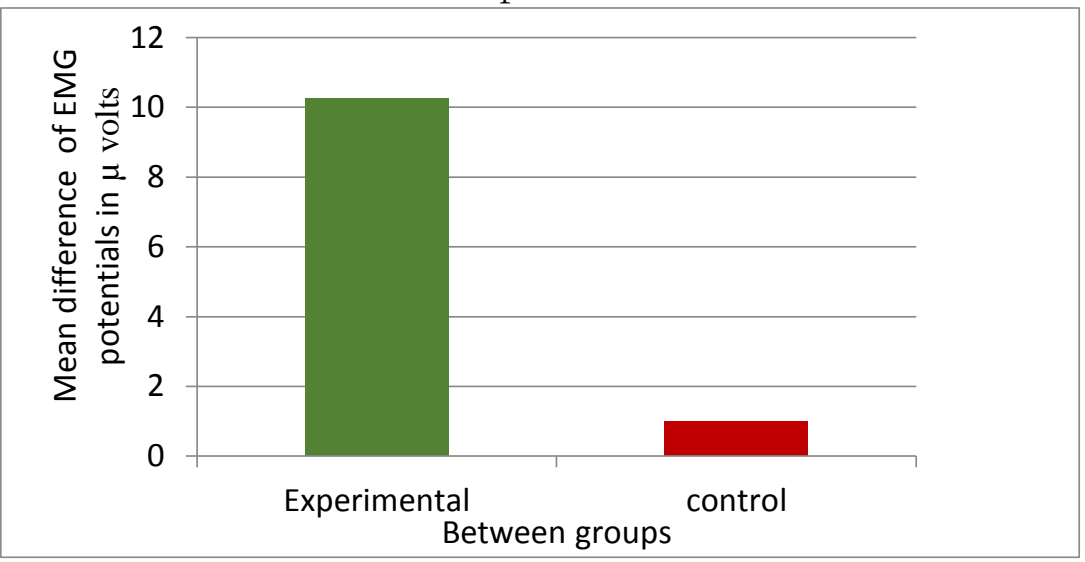

There was a significant difference between experimental group and control group in EMG potentials on hand function in subjects with stroke.

B \& BT: To compare the results of between the group of control $\&$ experimental group, unpaired ttest was selected. The p-value was 0.001; where the difference was considered very significant. The values of BEBT were improved in control group as well experimental group but the improvement was more in experimental group. Thus the null hypothesis is rejected and alternate hypothesis is accepted.

Chart: 6 Graphical representation of mean difference between experimental group and control group in B E BT:

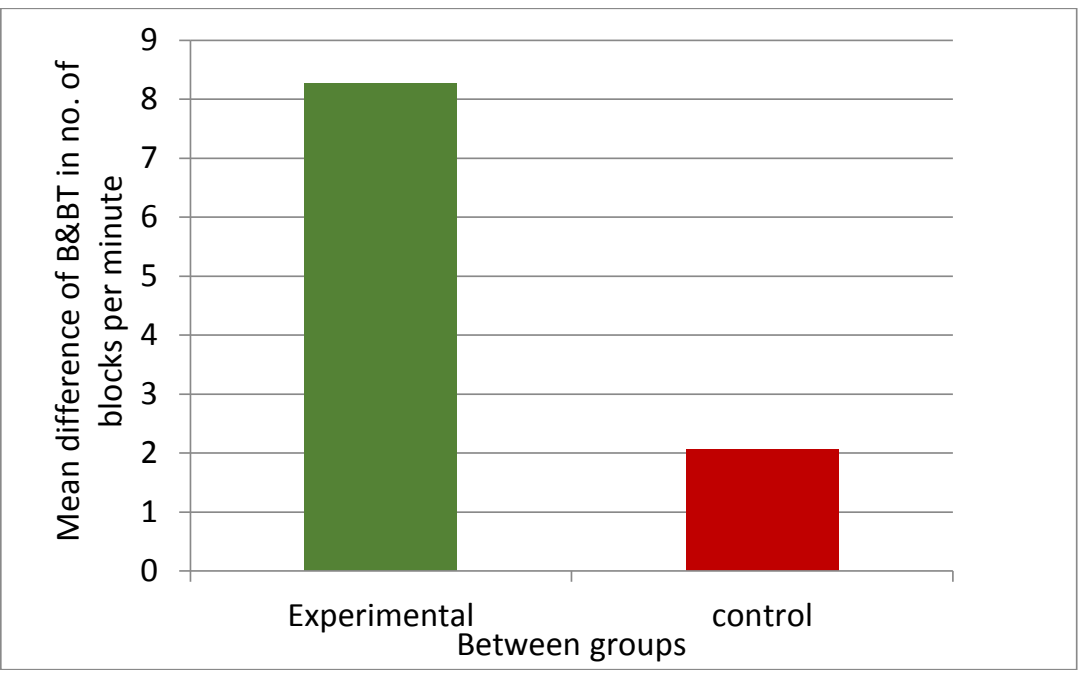


There was a significant difference between experimental group and control group in BEBT on hand function in subjects with stroke.

\section{DISCUSSION}

The results of the present study revealed that there was a significant difference in both control and experimental group which indicates that EMG biofeedback was effective in improving hand function in stroke subjects.

Rehabilitation of the upper extremity stroke poses a major challenge to patients who have sustained a physical therapy. In a review of studies on upperextremity recovery, Gowland stated that only $4 \%$ to $9 \%$ of patient's regained normal function, $23 \%$ to $43 \%$ regained some useful function, and $16 \%$ to $28 \%$ did not have return of any voluntary movement in the upper limb. One technique used to improve upper extremity movement following stroke is electromyographic (EMG) biofeedback. Knowledge of its efficacy is important for decisions related to patient care and the utilization of limited rehabilitation resources.

Armagan and colleagues in 2003 studied 27 patients assigned by RCT into EMG group and placebo-EMG group; both treatments were applied five times a week for a period of 20 days. In addition, the patients in both groups received an exercise program according to the Brunnstrom's neurophysiologic approach. Goniometric measurements for wrist extension scale for judging the performance of drinking from a glass, Brunnstrom's stages of recovery for hand, and surface EMG potentials were used for the clinical assessments. All patients were assessed before treatment and after 20 treatment sessions. The results showed that there were statistically significant improvements in all variables in both groups, but the improvements in active range of motion and surface EMG potentials were significantly greater in the EMG biofeedback group at the end of the treatment; which demonstrated the potential benefits of EMG biofeedback in conjunction with neurophysiologic rehabilitation technique in maximizing hand function in hemiplegic patients but the limitations of the study include duration of the treatment was small and sample size was also small and there was lack of long-term observation because outcomes were assessed only at discharge,

More recently study was conducted between January 2011- November 2011 by Maheshwari S Harishchandre, Singaravelan R M in College of Physiotherapy, Pravara Institute of Medical Sciences, Loni, Maharashtra State, Thirty participants of both genders, aged between 40-65 and those who met all the eligibility criteria were included. They were divided into two equal groups, Group A Study group received EMG Biofeedback along with conventional physiotherapy and Group B Control group received only conventional physiotherapy. Intervention given over a period of 4 weeks, baseline and post values were assessed by Voluntary Control Grading Scale and Action Research Arm test. The results of the study revealed that there was a significant difference between both the groups on improved hand voluntary control $(p<0.01)$ and improved hand function $(p<0.01)$ in hemiplegic stroke participants but the limitations of this present study focused on only in MCA stroke participants as well as only subacute stage hemiplegic participants and ARA test and voluntary control grading scale both outcome measures were depends on the condition, the severity of disability of participants.

In our study, EMG biofeedback was used, which enhances forced active movements of hemipaeritic limb promoting motor and functional recovery. In stroke subjects initiating wrist extension is often difficult for many patients because of the flexor synergy in the upper limb and are usually neglected when constituting individual rehabilitation due to its discouraging results in recovery, causing a social stigma but in our study EMG biofeedback training facilitates wrist extensor activity because paralysis of the finger extensors does not interfere with the interphalangeal joints, so the function was performed by the interossei and lumbricals but if it is in immobile then the contractures of these intrinsic muscles lead to hyperextension of this joints as well as flexion contracture of the metacarpophalangeal. EMG biofeedback training improves the functional ability of the hand and also and decreases the flexor spasticity also. Improvement was showed because of EMG Biofeedback and conventional physiotherapy altered the motor unit activity based on augmented audio \& visual feedback information.

Repetitive exercise may be critical to motor learning and it may drive brain reorganization by a process of motor learning. Functional recovery in the arm was found to be significantly better in the experimental group compared to control group and this could be attributed mainly to the repetitive stimulation of muscle activity in the arm and this technique allowed the participants to alter motor unit activity based on augmented audio $\mathcal{E}$ visual feedback information and provides a notion that some type of sensory feedback mechanism must reinforce voluntary effort 
These results agree with those from two recent studies, in which active repetitive motor training of hand and fingers had proven to be effective and the time spent on this protocol was a significant predictor of improvement in BEBT in both groups. But in both groups due to fatigue during 30 minutes of EMG intervention rest intervals were indulged and they varied from subject to subject based on individual's muscular endurance and motivation levels.

There is growing evidence that muscle weakness rather than spasticity plays a dominant role in impairment of active voluntary movements. Evidence from fore mentioned studies suggests that using EMG Biofeedback in combination with conventional physiotherapy may result in improvements in motor power beyond those of conventional physiotherapy alone.

\section{CONCLUSION}

EMG biofeedback had proven as one of the effective methods of management for stroke related disabilities. EMG biofeedback was based on the concept that retention persists even after withdrawal of sensory feedback as a result of sensory motor integration taking place. EMG biofeedback which enhances the forced active movements of hemipaeritic limb promotes motor and functional recovery by a process called motor relearning. It is a set of processes associated with practice or experience leading to permanent changes in capability for responding. Hence the study recommends that EMG biofeedback is most effective in improving hand function in stroke subjects.

\section{REFERENCES}

1. Warlow C, Sudlow C, Dennis M, Warlow C, Sudlow C, Dennis M, et al. Stroke. Lancet. 2003; 362(9391):1211-24.

2. Strong K, Mathers C, Bonita R. Preventing stroke: saves lives around the world. Lancet Neurolgy. 2007; 6(2): 182-7.
3. World Health Organization. The economic impact of chronic diseases in preventing chronic diseases: a vital investment. World Health Organization, Geneva; 2005.

4. Bary-y-Rita. Recovery of functions: Theoretical considerations for brain rehabilitation.1980.

5. Heller A, Wade DT. Arm function after stroke: measurement and recovery over the first three month. J Neuro Neurosurgery Psychiatry.1987; 50(6):714-719.

6. Nakayama $H$, Jorgensen S, Raaschou HO, Oslen TS. Recocery of upper extremity function in stroke patients: the Copenhagen stroke study. Arch Phys Med Rehabili. 1994; 75(4):394-8

7. Kwakkel G, Kllen B, Vander Ground J, prevo AJ. Probability of regaining dexterity in the flaccid upper limb. Stroke. 2003; 34(9):2181-6

8. Davies PM. Steps to follow. $2^{\text {nd }}$ ed;1985.

9. Carr J Shephered R. Neurological rehabilitation: optimizing motor performance. $2^{\text {nd }} e d ; 2010$.

10. Taub E, Crago JE, Burgio LD, et al. An operant approach to rehabilitation medicine: overcoming learned mouse by shaping .J Exp anal Behav. 1994;61(2):281-93.

11. Carey LM. Somatosensory loss after stroke. Crit Rev Phy Rehabili Med. 1995;7(1): 51-91

12. Lippert j,Graef S, uhdeI. Liedner O, Weiller c, Training induced changes in motor cortex representation in stroke patients. Acte Neurol Scand 2000; 101(5):321-6.

13. taub E, Berman A. Movement and learning in the absence of sensory feedback.In: Freedman S,rd. Thw neuropsychology of spatialy oriented behavior. Homewood Dorsey press.1968;173192.

14. Nichols-Larsen DS, Clark PC, Zeringue A, Greenspan A, Blanton S. Factors influencing stroke survivors quality of life during subacute recovery. Stroke. 2005;36(7):1480-1484.

15. Giggins et al. Biofeedback in rehabilitation. Journal of NeuroEngineering and Rehabilitation 2013, 10:60

\section{Citation}

S. Sethana, V. Sri Kumari, \& K. Madhavi. (2014). THE EFFECTIVENESS OF EMG BIOFEEDBACK ON HAND FUNCTION IN SUBJECTS WITH STROKE. International Journal of Physiotherapy, 1(4), 178-186. 